\title{
Recomendaciones para el manejo de pacientes con COVID19 en el perioperatorio
}

\author{
Comité Científico Sociedad de Anestesiología de Chile: Aranda F, Aliste J, Altermatt F, \\ Alvarez JP, Bernucci F, Cabrera MC, Carrasco E, De la Fuente R, Egaña Jl, Lacassie H, \\ Merino W, Penna A, Torres D.
}

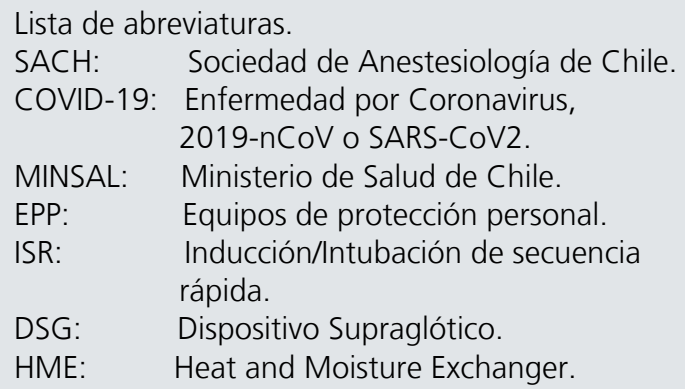

\section{Desarrollo de la Recomendación Clínica (RC)}

\subsection{Introducción}

En diciembre de 2019, una serie de casos de neumonía de causa desconocida surgieron en Wuhan, Hubei, China, con presentaciones clínicas muy parecidas a la neumonía viral. El análisis de secuenciación profunda de muestras del tracto respiratorio inferior indicó un nuevo coronavirus, que se denominó nuevo coronavirus 2019 (2019-nCoV o SARS-CoV2) [15]. La propagación del virus ha sido extremadamente rápida, tanto así que el 11 de marzo de 2020 y luego de más de 118.000 infectados en 114 países con 4.300 fallecidos a esa fecha, la organización mundial de la salud declaró a la enfermedad COVID-19, causada por 2019-nCoV, como una pandemia[16].

Las potenciales repercusiones de la enfermedad han llevado a las organizaciones de salud a nivel mundial, regional y locales a implementar una serie de medidas para hacer frente al COVID-19 e intentar disminuir su impacto. Es en este contexto que la SACH ha decidido elaborar estas recomendaciones para el manejo perioperatorio de los pacientes sospechosos y/o portadores de la enfermedad. La necesidad de estas recomendaciones queda clara si se considera que el personal de salud es uno de los que más se ha visto afectado, en especial en etapas tempranas de la pandemia y es, por su labor, un potencial agente trasmisor de gran capacidad.

Estas recomendaciones vienen a complementar las normativas generales impuestas por las autoridades sanitarias del país en un aspecto particular como es el período perioperatorio. Es necesario hacer hincapié que, desde el punto de vista individual, para lograr un correcto actuar frente al COVID-19 se deben cumplir tanto las normas generales (aseo frecuente de manos, limpieza de superficies, distanciamiento social) como las específicas aquí presentadas. Es necesario, por lo tanto, que cada miembro de la sociedad conozca todos los protocolos.

La SACH espera que estas recomendaciones contribuyan a establecer medidas eficaces para disminuir la propagación del COVID-19 y puedan servir de guía a las autoridades pertinentes. Por lo mismo es que estarán, a partir de su publicación, en constante revisión por el mismo comité que las creó, para evaluar eventuales modificaciones o actualizaciones.

\subsection{Objetivo General}

Recomendar cómo enfrentar anestésicamente a un paciente en el perioperatorio con sospecha o con confirmación de estar infectado por el COVID-19 con la finalidad de disminuir el riesgo de infección del personal de salud, otros pacientes y la comunidad.

\subsection{Escenarios clínicos y condiciones de aplica- ción}

Esta recomendación se aplica en el contexto del manejo perioperatorio de un paciente con sospecha o con diagnóstico de infección COVID-19. Por ello, abarca desde la evaluación inicial del paciente en el lugar en el que estuviese hospitalizado hasta que es devuelto a la unidad donde realizará el postoperatorio tardío. Del mismo modo, está destinada a todo procedimiento que va a requerir un apoyo anestésico dentro o fuera de pabellón. Esto puede ir desde el

Fecha de presentación de la RC. 16 de marzo de 2020. 
apoyo a una intubación de la vía aérea en cualquier unidad hospitalaria, una anestesia general de mayor complejidad o la administración de otras formas de anestesia (sedación, anestesia regional) en pacientes con diagnóstico o sospecha de infección. El enfoque evidentemente está orientado al correcto uso de los EPP, sobretodo con respecto al manejo de la vía aérea en todo tipo de procedimiento, es decir no sólo intubación orotraqueal.

\section{Métodos}

\subsection{Métodos de consenso utilizados para la for- mulación de recomendaciones}

En la formulación de la RC se establecieron controversias con respecto a:

- La validez de estudios y guías publicadas para el contexto clínico.

- La condiciones de aplicabilidad o el impacto esperado de las recomendaciones.

- Incorporación de nuevos artículos en la búsqueda de evidencia o bibliografía.

- Las evidencias encontradas resulten contradictorias o las condiciones locales no hagan aplicable la recomendación.

Para resolver estas diferencias aplicaron métodos de consenso:

- Representatividad: Todos los autores o al menos tres de ellos participaron de la resolución de la controversia.

- Todos los miembros del grupo a cargo de la elaboración del documento dispusieron del mismo nivel de información básica para la toma de decisiones.

\subsection{Grados de evidencia y niveles de recomen- dación utilizados en la RCs}

Para esta recomendación la evidencia disponible descansa principalmente en opinión de expertos y lo presentado a continuación tiene ese grado de evidencia dado fundamentalmente por el número de publicaciones (referencias) y por lo reciente del cuadro de rápida expansión.

Según la evolución epidemiología de la pandemia y la aparición de nueva evidencia se realizará actualizaciones de este documento según se necesario.

\subsection{Consideraciones ético-sociales}

A través de este documento, la SACH desea entregar una herramienta que permita enfrentar de mejor manera a pacientes con sospecha o diagnóstico de COVID-19 que requieren manejo de la vía aérea, ya sea para soporte ventilatorio o en un escenario perioperatorio. Se pone a disposición de los pacientes, autoridades sanitarias y anestesiólogos(as) del país, información pertinente para confrontar los nuevos desafíos que esta pandemia presenta a la Anestesiología. De esta manera, la SACH cumple con su propósito, su responsabilidad ética y compromiso con el país, de aportar antecedentes para una mejor atención de la comunidad.

\section{Recomendaciones}

\subsection{Condiciones de traslado a pabellón y Preope- ratorio}

\subsubsection{Organización del procedimiento}

Recomendamos:

- Se recomienda encarecidamente a los anestesiólogos que hagan los mayores esfuerzos para tener información actualizada de las recomendaciones de cada centro en particular, junto con conocer los espacios físicos y los elementos que van a disponer para enfrentar cada caso sospechoso o confirmado de COVID-19.

- Un plan de acción del equipo de anestesia preparado y verbalizado entre los profesionales con una asignación clara de tareas respectivas.

- Implementos, fármacos y equipamiento de anestesia deben estar preparados con anticipación.

- El entrenamiento del personal previamente a que aparezca el primer caso para evitar errores.

- La programación de la intervención quirúrgica debe, en lo posible, ser hecha en los horarios de menor circulación de personal.

- La eliminación de los desechos contaminados tras el haber realizado el procedimiento debe seguir los lineamientos del establecimiento.

\subsubsection{Equipamiento del personal de salud en el pabellón}

Frente a un paciente quirúrgico con sospecha o con confirmación de estar infectado por el COVID-19, el personal implicado en el procedimiento debe poseer sus EPP según las reglas para COVID-19 establecidas por el MINSAL o equipo de Infectología del establecimiento. Este equipamiento se compone de:

- Una máscara de protección (idealmente una N95 - FFP2 o equivalentes) para el personal de salud.

- Protección ocular.

- Una bata quirúrgica con mangas y puños impermeables. 
- Guante no estériles, bien adosados a la piel (látex o de nitrilo).

- Recomendamos el uso de doble guante para la persona que maneja la vía aérea.

Recomendamos:

- Entrenamiento del personal para los procedimientos de vestir y desvestir, para evitar potenciales errores y contaminación.

- Lavado de manos después del retiro y eliminación del EPP.

\section{Comentario:}

El riesgo de máxima contaminación del clínico ocurre en el momento de desvestirse y eliminar el equipamiento al final del procedimiento, lo cual debe estar supervisado por una persona preparada.

\subsubsection{Preparación del paciente y procedimiento de apertura del pabellón}

El traslado de un paciente sospechoso o confirmado de infección por COVID-19 a la sala de operaciones y su retorno a la sala de aislamiento recomendamos:

- No llevar al paciente a las áreas de espera o de la unidad preoperatoria. Se debe asignar un quirófano determinado para la atención de este tipo de pacientes y colocar letreros en las puertas para minimizar la exposición del personal.

- Los pasillos y ascensores deben estar despejados de cualquier persona.

- El paciente debe portar una máscara de tipo quirúrgica durante el traslado hacia la sala de operaciones.

- Las personas encargadas del traslado y de la recepción en pabellón deben portar su EPP contando con una máscara de tipo quirúrgica.

- Corroborar que, de ser posible, la ventilación del pabellón esté funcionando a presión negativa y evitar la presión positiva.

- Las drogas anestésicas e insumos a utilizar deben estar preparados previo al ingreso del paciente al pabellón para minimizar la apertura de los cajones de los carros.

- Todo el personal que no maneja la vía aérea al interior del pabellón debe portar su EPP contando con máscara de tipo quirúrgica.

- La desinfección de las manos por lavado de manos con agua y jabón de Clorhexidina es indispensable antes de entrar en contacto con el paciente y posterior a ello.

- Se debe limitar el número de personas a cargo del paciente al mínimo necesario, idealmente sin salida ni intercambio de personal durante todo lo que dure el procedimiento.

3.2. Procedimiento de inducción anestésica, manejo de vía aérea y extubación

\subsubsection{Generalidades}

Recomendamos:

- Designar al operador más experto para realizar los procedimientos.

- De ser posible, evite intubaciones con fibrobroncoscopio ya que tienen más riesgo de producir aerosoles.

- Es recomendable utilizar tubos orotraqueales (con cuff) y limitar los DSG solo para situaciones de rescate de vía aérea.

- La monitorización a elegir está dada por la situación del paciente, la cirugía a realizar y la técnica anestésica elegida, como en cualquier persona sometida a anestesia (ver recomendaciones SACH).

- Se debe minimizar las intervenciones que favorecen la liberación de partículas aerosolizadas, ventilación con máscara facial o máscara laríngea. Se hace indispensable identificar a aquellos pacientes con predictores de mayor complejidad y preparar una estrategia de manejo de vía aérea de modo que el primer intento sea el mejor intento.

\section{Comentario:}

La transmisión de COVID-19 es por inhalación de gotitas, contacto con personas infectadas especialmente a menos de 2 metros y por contacto con mucosas. Los métodos aparentemente efectivos de protección son: EPP, métodos simples de descontaminación, agua, jabón y alcohol. Se recomienda uso estricto de EPP.

El aislamiento mínimo es el de gotitas y contacto. Debe haber espacio físico para instalar y desechar el EPP en forma segura.

\subsubsection{Instrumentalización de la vía aérea}

Recomendamos:

- Asegúrese de estar usando protección adecuada (ver 5.1.2). Utilice doble guante ya que esto permite cubrir fácilmente, con el primer par de guantes, la hoja del laringoscopio una vez realizada la intubación y fijación del tubo.

- Preoxigene al paciente por 5 minutos a $\mathrm{FiO}_{2}=$ $100 \%$ y prepárese para una ISR. De ser posible utilice dosis más bien altas de bloqueador neuromuscular para prevenir lo más posible que el pa- 
ciente tosa durante el procedimiento.

- En lo posible, prefiera el uso de videolaringoscopio y tubo con conductor para minimizar los intentos de intubación y aumentar la distancia entre el paciente y el operador.

- Realizar una ISR. De ser posible evite la ventilación manual con bolsa. Si la ventilación manual es indispensable, aplique volúmenes corrientes bajos.

- Coloque un filtro HME entre la pieza en Y del circuito de respiración y la máscara del paciente, el tubo endotraqueal o la máscara laríngea (Figura 1).

- Alternativamente, para pacientes pediátricos $u$ otros pacientes en los que el espacio muerto adicional o el peso del filtro pueden ser problemáticos, el filtro HME debe colocarse en el extremo espiratorio del circuito de respiración corrugado antes de que el gas espirado ingrese a la máquina de anestesia.

- La toma de muestreo de gases también debe estar protegida por un filtro HME, y los gases que salen del analizador de gases. Idealmente deben ser eliminados y no se debe permitir que regresen al aire de la habitación.

- Si está disponible, use un sistema de aspiración cerrado durante la succión de la vía aérea.

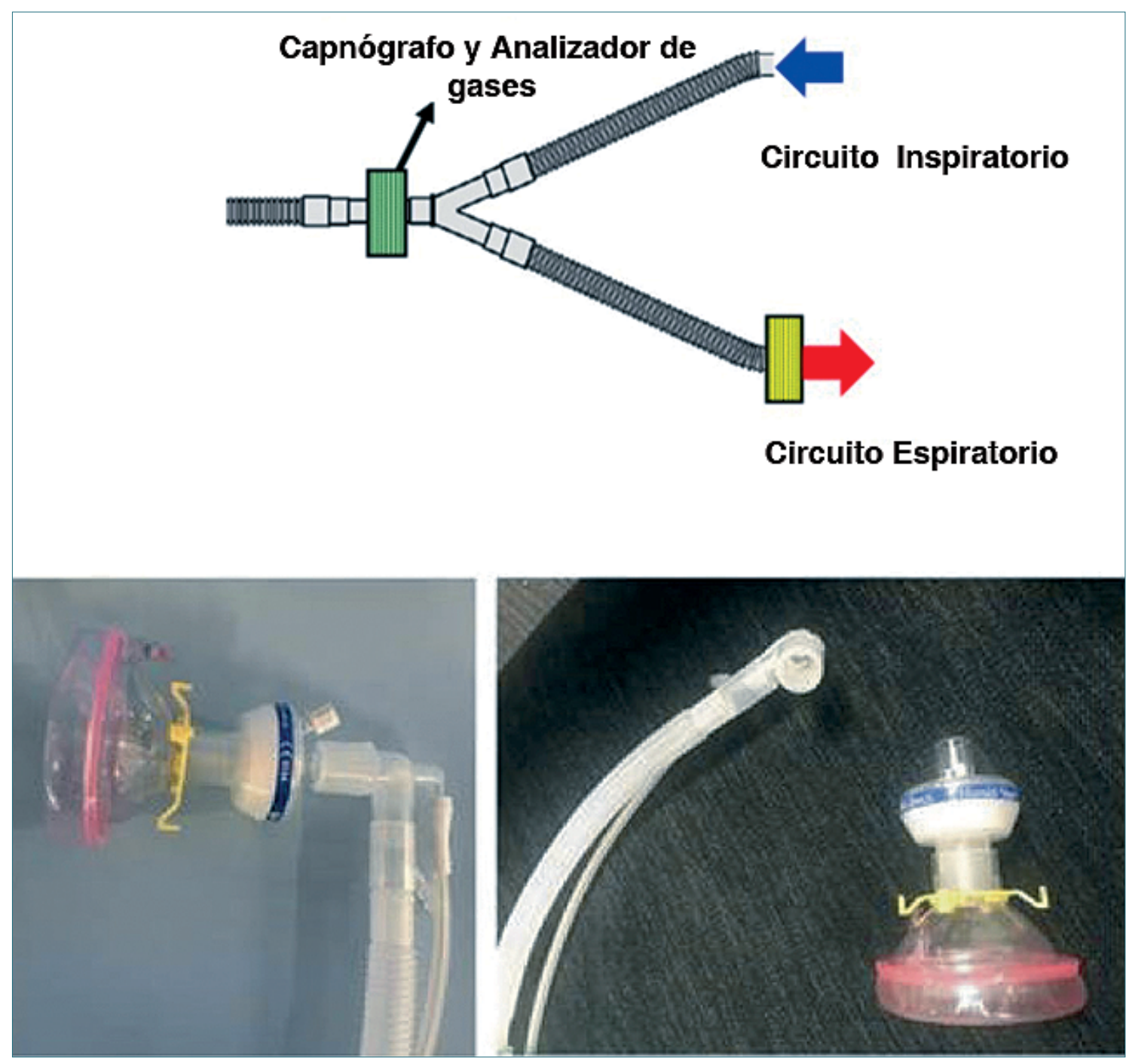

Figura 1. Extraído de ASA. 
- De ser posible conectar la máquina de anestesia a la red de eliminación de gases de deshecho, de no ser posible, utilizar flujos bajos.

- Vuelva a envolver el laringoscopio inmediatamente después de la intubación (técnica de doble guante). Selle TODOS los equipos de vía aérea usados en una bolsa de plástico.

- Considere desechar la trampa de agua (defend) una vez terminado el procedimiento.

- El paciente debe recuperarse en la sala de operaciones o transferirse a una sala de aislamiento de infecciones transmitidas por el aire.

- Después de que el paciente haya salido del quirófano, deje transcurrir el mayor tiempo posible antes de la atención del siguiente paciente (para eliminar la contaminación infecciosa en el aire).

- Después del caso, limpie y desinfecte las superficies de alto contacto en la máquina de anestesia y el área de trabajo de anestesia con un desinfectante hospitalario adecuado.

- Si se utilizan dispositivos como el ultrasonido en el punto de atención:

- Se debe usar una cubierta plástica larga para la unidad de ultrasonido y el cable para minimizar la contaminación del equipo.

- Las partes no esenciales del carro de ultrasonido pueden cubrirse para minimizar la exposición a las gotitas.

- Después de quitar el equipo de protección, recuerde evitar tocarse el cabello o la cara antes de lavarse las manos.

\subsection{Conducta para anestesia regional}

Recomendamos:

- Mantener la mascarilla quirúrgica en pacientes que se sometan a anestesia regional.

- Verificar el recuento de plaquetas antes de insertar epidural o espinal, y posiblemente antes de la extracción de catéter epidural (un tercio de los infectados se constató una caída de las plaquetas bajo 150.000).

- Minimizar entrada de personal a pieza de paciente utilizando métodos automáticos de analgesia de parto de estar disponibles y debidamente protocolizados en orden de minimizar los contactos con la paciente.

\subsection{Manejo posterior al procedimiento}

Una vez terminado el procedimiento quirúrgico se recomienda:

- Los guantes deben quitarse inmediatamente y realizar una desinfección de manos por fricción hidro-alcohólica ANTES de desvestirse.

- Después de desvestirse, el personal debe evitar el contacto de sus manos con su cabeza o cuerpo antes de realizar un segundo lavado de manos.

- La eliminación de los EPP al final del procedimiento debe ser realizada evitando tocar las zonas contaminadas.

- El material anestésico se debe limpiar con productos detergentes descontaminantes adecuados. Esto debe ser realizado por personal clínico, no de aseo habitual.

- Avisar al servicio de destino que se procederá al traslado del paciente e iniciarlo cuando el servicio al cual será llevado confirme que está en condiciones de recibirlo.

- Coordinar con el sistema de seguridad del centro hospitalario el traslado de modo de lograr la máxima fluidez posible (uso de ascensores, evitar pasillos de riesgo, etc.).

- En caso de decidir extubar el paciente, este debe hacer el período de post anestesia en pabellón y ser trasladado a su pieza con una mascarilla quirúrgica y según el protocolo establecido en puntos anteriores.

- Una vez realizado el traslado la eliminación de los EPP debe realizarse una vez entregado el paciente y según el protocolo de retiro de EPP.

- El monitoreo de traslado de los pacientes se debe realizar según las necesidades que estime el anestesiólogo tratante y realizar la protección, limpieza o desecho de lo utilizado según los protocolos establecidos.

- Avisar al personal de aseo para realizar la limpieza del pabellón y material anestésico según el protocolo institucional de IIAS.

- El tiempo estimado entre casos es de una hora, lo que permite, traslado, aseo y preparación de próximo caso. 


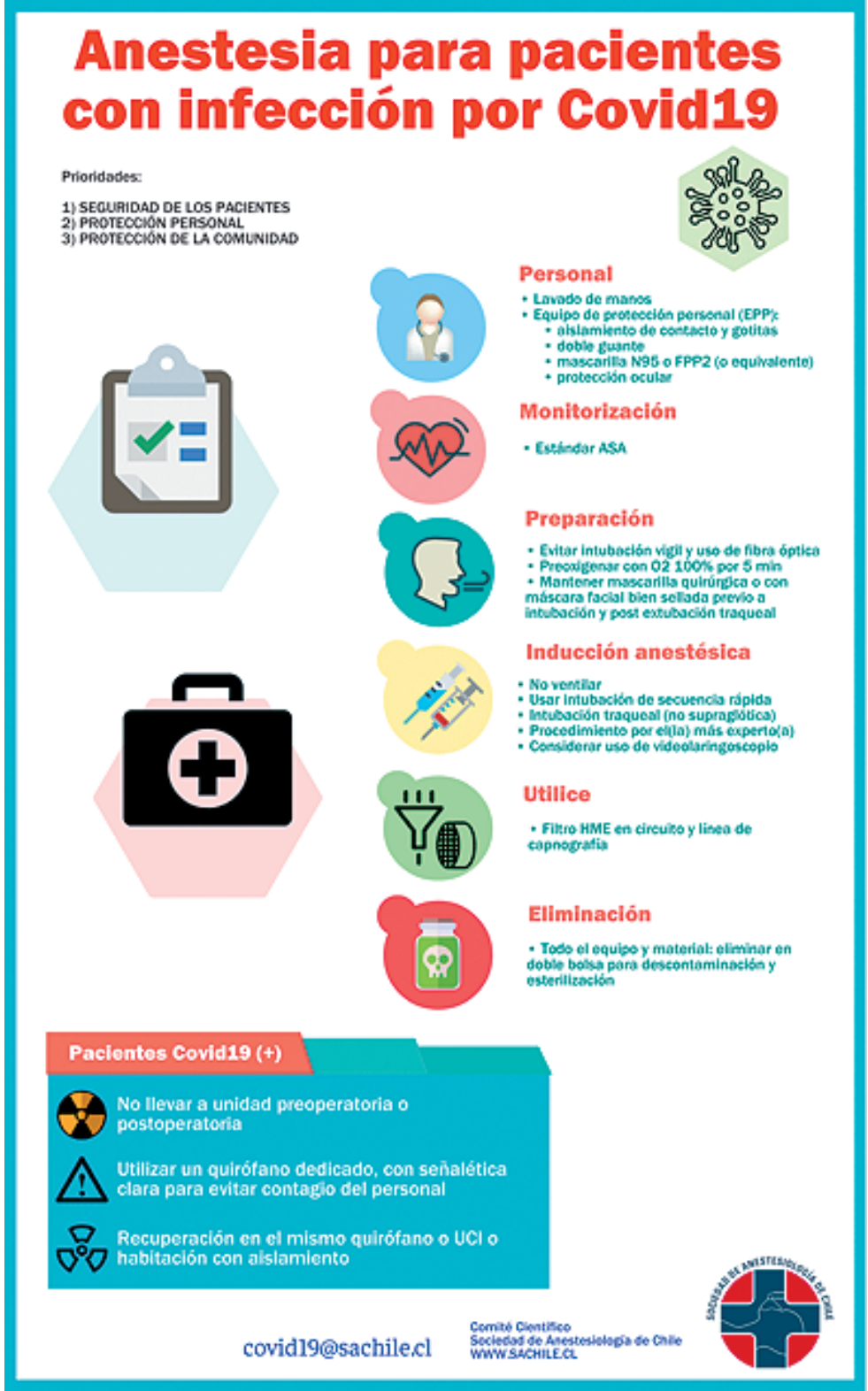

\section{Referencias}

1. https://www.anesthesia.utoronto.ca/news/coronavirus-andsafety-precautions

2. Lian Kah. Lin Stella Ang, Theng wai Foong Bryan Su Wei Ng. What we do when a COVID-19 patient needs an operation: operating room preparation and guidance. Can J Anesth; 2020. https://doi.org/10.1007/s12630020-01617-4.

3. Wax RS, Christian MD. Practical recommendations for critical care and anesthesiology teams caring for novel coronavirus (2019-nCoV) patients. Can J Anesth 2020; DOI: https:// doi.org/10.1007/s12630-02001591-x.

4. Government of Singapore. Coronavirus disease 2019: cases in Singapore. Available from 
URL: https://www.gov.sg/article/ covid-19-cases-in-singapore.

5. World Health Organization. Coronavirus disease (COVID-2019) situation reports. Available URL:https://www.who.int/emergencies/diseases/novel-coronavirus-2019/situation-reports

6. Galeano Carlos, Trujillo A. Recomendaciones para el manejo perioperatorio de pacientes con infección o sospecha de infección por COVID -19. Sociedad colombiana de anestesiología y reanimación.

7. Peng PW, Ho PL, Hota SS. Outbreak of a new coronavirus: what anaesthetists should know. Br J Anaesth. 2020 Feb; https://doi. org/10.1016/j.bja.2020.02.008 PMID:32115186

8. World Federation of Societies of Anesthesiologists. Coronavirus - guidance for anaesthesia and perioperative care providers.

9. SFAR. Propositions pour la prise en charge anesthésique d'un patient suspect ou infecté á Coronavirus COVID-19. 13 mars 2020.

10. Zhao S, Ling $K$, Yan $H$, Zhong L, Peng $X, Y$ Yoo $S$, et al. Anesthetic
Management of Patients With

Suspected or Confirmed 2019

Novel Coronavirus Infection

During Emergency Proce-

dures. J Cardiothorac Vasc

Anesth. 2020 Feb; https://doi.

org/10.1053/j.jvca.2020.02.039

PMID:32178954

11. Best practice recomendations for anesthesiologists during intubation of patients with coronavirus. Department of Anesthesiology and Pain Medicine. University of Toronto. January 25, 2020.

12. Zuo MZ, Huang $Y G, M a W H$, Xue ZG, Zhang JQ, Gong $\mathrm{YH}$, et al.; Chinese Society of Anesthesiology Task Force on Airway Management. Expert recommendations for tracheal intubation in critically ill. Chin Med Sci J. 2020 Feb; https:// doi.org/10.24920/003724 PMID:32102726

13. WFSA. Coronavirus-guidance for ananesthesia and perioperative providers. March 2020.

14. APSF (Anesthesia Patient Safety Foundation). Perioperative Considerations for the 2019 Novel Coronavirus (COVID-19). February 12, 2020.
15. Huang $C$, Wang $Y$, Li $X$, Ren L, Zhao J, Hu Y, et al. Clinical features of patients infected with 2019 novel coronavirus in Wuhan, China. Lancet. 2020 Feb;395(10223):497-506. https://doi.org/10.1016/ s0140-6736(20)30183-5 PMID:31986264

16. https://www.who.int/es/dg/ speeches/detail/who-directorgeneral-s-opening-remarks-atthe-media-briefing-on-covid19-11-march-2020

17. https://www.esahq.org/esanews/covid-19-airway-management/

18. https://www.asa.org.au/wordpress/wp-content/uploads/News/ eNews/covid-19/ASA_airway_ management.pdf

19. https://www.asahq.org/aboutasa/governance-and-committees/asa-committees/committeeon-occupational-health/coronavirus/

20. Philip W. H. Peng, Pak-Leung Ho and Susy S. Hota. Outbreak of a new coronavirus: what anaesthetists should know. British Journal of Anesthesia. 2020 Feb; https://doi.org/10.1016/j. bja.2020.02.008 